\title{
Welche Arbeitszeitpolitik? Ein neues Verhältnis von Zeitökonomie und Zeitpolitik
}

\begin{abstract}
Der Wandel der Arbeitszeitorganisation - zugespitzt: von der Stechuhr zur Vertrauensarbeitszeit - ist weit mehr als nur eine Veränderung von Organisationsformen. Die Stechuhr ist ein Symbol für die betriebliche Herrschaft über die Zeit. Dagegen steht die Vertrauensarbeitszeit für einen neuen Zusammenhang von Zeitökonomie und Zeitpolitik. Das richtige Ergebnis und der richtige Zeitpunkt werden mehr und mehr zum zentralen Maß der Zeitökonomie, die politischen Organe werden entmachtet, die Individuen ermächtigt - und zugleich überfordert. Dass dieser Entwicklung mit einer „neuen Arbeits(zeit)politik“ Rechnung getragen werden muss, ist klar - aber welche Arbeitszeitpolitik ist heute nötig und möglich?
\end{abstract}

\section{Einleitung}

Unter Stichworten wie „Flexibilisierung“ oder „Erosion der Normalarbeitszeit“"werden seit rund zwei Jahrzehnten tief greifende Umbrüche in der Arbeitszeitorganisation diskutiert. Die beobachtbaren Entwicklungen, die damit beschrieben werden, sind äußerst vielgestaltig, uneinheitlich und kaum auf einen Nenner zu bringen. Fragt man aber nach so etwas wie den zentralen Prinzipien der Arbeitszeitorganisation, dann lassen sich - in der Debatte und in der realen Entwicklung - zwei Pole des anhaltenden Umbruchs ausmachen: Den einen Pol bildet die durch die Stechuhr symbolisierte Arbeitszeitregulierung im Fordismus-Taylorismus. Deren wesentliches Merkmal ist die kollektive Regulierung des Verhältnisses von Arbeitszeit und privater Zeit durch standardisierte, jeweils für größere Gruppen gültige, in Dauer und Lage individuell nicht variierbare Arbeitszeiten. Am anderen Pol steht die Vertrauensarbeitszeit, also eine Arbeitszeitorganisation, in der die Betriebe komplett auf die Erfassung und Kontrolle der Arbeitszeit verzichten und die konkrete Ausgestaltung der Arbeitszeit den Beschäftigten selbst überlassen. Beide Pole symbolisieren weit mehr als nur (mögliche) Anfangs- und Endpunkte einer Entwicklung oder unterschiedliche institutionelle Formen der Arbeitszeitorganisation sie sind diametral unterschiedliche Prinzipien, wie Arbeitskraft genutzt und ihr Einsatz organisiert wird (Haipeter et al. 2002). Der historische Prozess der Veränderung der Arbeitszeitorganisation, der gegenwärtig unter den Stichworten Flexibilisierung, aber auch Retaylorisierung thematisiert wird, ist durch die Gleichzeitigkeit und das Nebeneinander beider Prinzipien gekennzeichnet. Diesen Prozess zu interpretieren und in seiner historischen Ausrichtung zu bestimmen, erscheint uns deshalb ohne eine Auseinandersetzung mit den darin erkennbaren grundlegenden Organisationsprinzipien nicht möglich.

Diese Unterscheidung von Prinzipien oder Logiken der Arbeitszeitorganisation spielt eine entscheidende Rolle für jede Antwort auf unsere Eingangsfrage: Welche Arbeitszeitpolitik ist heute nötig und möglich? Wir spitzen unsere Überlegungen bewusst auf die angesprochenen Pole der Arbeitszeitorganisation zu, um die prinzipielle Dimension des Themas herauszuarbeiten. Ausgehend von der Frage, was Betriebsräte und Gewerkschaften gegen Vertrauensarbeitszeit haben (können), suchen wir Antworten vor allem in zwei Richtungen: erstens im Zusammenhang von (gewerkschaftlicher) Arbeitszeitpolitik und dem Wandel betrieblicher Herrschaft; zweitens im sich verändernden Verhältnis von Zeitökonomie und Zeitpolitik. Abschließend unternehmen wir dann den Versuch, auf der Basis dieser Überlegungen erste Antworten auf die Frage zu geben: Welche Arbeitszeitpolitik?

\section{Von der Stechuhr zur Vertrauensarbeitszeit - und zurück?}

Über die Verbreitung der Vertrauensarbeitszeit ist nach wie vor nicht viel Gesichertes bekannt. Dies liegt nicht zuletzt daran, dass darunter alles Mögliche verstanden wird und dass entsprechende Angaben teilweise interessengeleitet sind (Haipeter et al. 2002). Mit den Daten der ISO-Arbeitszeitberichterstattung lässt sich schätzen, dass bundesweit rund $8 \%$ aller Beschäftigten ihre Arbeitzeit formal gesehen frei bestimmen - mit hoch qualifizierten Angestellten an der Spitze (22\%) und Facharbeitern (3\%) als Schlusslicht (Bauer et al. 2004). Mit $32 \%$ deutlich größer ist der Anteil der Beschäftigten, die unter den weiter gefassten Bedingungen „variabel selbst gesteuerter" Arbeitszeit arbeiten (ebd.). Kritiker wie Befürworter sind sich zwar relativ einig in der Einschätzung, dass Vertrauensarbeitszeit in Zukunft eine eher wachsende Rolle spielen wird. Empirische Befunde aus Expertengesprächen kommen

Nick Kratzer, Dr., Sozialforscher am Institut
für Sozialwissenschaftliche Forschung e. V.
(ISF) München. Arbeitsschwerpunkte:
Betriebliche Reorganisations- und Rationali-
sierungsstrategien, Entgrenzung von Arbeit
und Leben, Flexibilisierung und Subjektivie-
rung von Arbeit, partizipatives Gesund-
heitsmanagement und Arbeitsschutz.
e-mail: nick.kratzer@isf-muenchen.de
Dieter Sauer, Prof. Dr., Sozialforscher am
Institut für Sozialwissenschaftliche
Forschung e. V. (ISF) München und Honorar-
professor an der Friedrich-Schiller-Univer-
sität Jena. Arbeitsschwerpunkte: Betriebliche
Reorganisations- und Rationalisierungs-
strategien, Entgrenzung von Arbeit und
Leben, Flexibilisierung, Subjektivierung und
Vermarktlichung von Arbeit.
e-mail: dieter.sauer@isf-muenchen.de

Nick Kratzer, Dr., Sozialforscher am Institut für Sozia/wissenschaftliche Forschung e. V. (ISF) München. Arbeitsschwerpunkte: Betriebliche Reorganisations- und Rationalisierungsstrategien, Entgrenzung von Arbeit und Leben, Flexibilisierung und Subjektivierung von Arbeit, partizipatives Gesundheitsmanagement und Arbeitsschutz. e-mail: nick.kratzer@isf-muenchen.de Dieter Sauer, Prof. Dr., Sozialforscher am Institut für Sozialwissenschaftliche Forschung e. V. (ISF) München und Honorarprofessor an der Friedrich-Schiller-Universität Jena. Arbeitsschwerpunkte: Betriebliche Reorganisations- und Rationalisierungse-mail: dieter.sauer@isf-muenchen.de 
aber eher zu dem Schluss, dass sich kein massiver Trend in Richtung Vertrauensarbeitszeit ausmachen lässt (Böhm et al. 2004). Dies hat - und auch das sehen Kritiker wie Befürworter wiederum ähnlich nicht zuletzt mit Widerständen und Befürchtungen aus verschiedenen Richtungen zu tun.

Der Arbeitszeitberater Andreas Hoff vertritt die Ansicht, dass Modelle von Vertrauensarbeitszeit „in die Lücke zwischen die klassischen Pole Arbeitgeber und Arbeitnehmer hineinpassen und (...) dazu beitragen, dass sich diese immer weiter schließt" (Hoff o. J.). Es geht darum, dass die Arbeitgeber Kontrolle durch Vertrauen ersetzen (sollen) - was ja zunächst ganz gut klingt - und dass mit der Vertrauensarbeitszeit die Grenze zwischen Arbeitgeber und Arbeitnehmer aufgehoben wird. Warum könnten nun Betriebsräte und Gewerkschaften etwas dagegen haben? An die Stelle von Kontrolle tritt doch demzufolge nicht nur eine selbstständig organisierte, sondern sogar eine selbstbestimmte Gestaltung der Arbeitszeit. Und in der Idee, Arbeitnehmer zu Arbeitgebern zu machen, ist zunächst auch kein Anlass für Kritik erkennbar.

Dass Betriebsräte und Gewerkschaften in der Praxis etwas dagegen haben, hat zunächst etwas mit Erfahrungswerten zu tun: Die Faustregel der bisherigen Erfahrung ist, dass die Beschäftigten umso länger arbeiten, je mehr Freiheiten sie in der Arbeit haben (Kratzer et al. 2005; Bauer et al. 2004). ${ }^{1}$ Vertrauensarbeitszeit oder andere Formen flexibler Arbeitszeit führen also gewöhnlich zur Arbeitszeitverlängerung. Und sie haben natürlich auch deshalb etwas dagegen, weil ihr Einfluss auf die Arbeits(zeit)gestaltung durch solche Formen der Arbeitszeitorganisation schwindet.

So verständlich diese Befürchtungen sind, sie erklären nicht, warum eine Rückkehr zu Regulierungsformen diskutiert und gefordert wird, die jahrzehntelang bekämpft wurden. Damals war die Rede vom „Terror der Stechuhr“, jetzt wird - zugespitzt gesagt - die Rückkehr der Stechuhr gefordert. Was - außer schlechten Erfahrungen und Befürchtungen für die Zukunft - liegt hinter diesem Sinneswandel?

\section{Zeitpolitik - Herrschaft über die Arbeitszeit- Grenzen}

Die Frage nach der betrieblichen Organisation und Regulierung von Zeit ist immer auch eine Frage nach Herrschaft. Zeit ist das gilt ganz generell - eine zentrale Dimension von Herrschaft. Zeit ist zum einen Objekt, also Gegenstand von Herrschaft: Herrschaft ist immer auch Herrschaft über die Zeit anderer, über deren Dauer, deren Lage und deren Nutzung. Und zum anderen ist Zeit ein Medium von Herrschaft, was man vielleicht dann am deutlichsten merkt, wenn man durch Entzug der Verfügungsgewalt über Zeit oder, im Gegenteil, durch die unfreiwillige Zuweisung von zuviel Zeit bestraft wird, also im „Freiheitsentzug" im Gefängnis, beim Nachsitzen in der Schule oder bei Zeitstrafen im Sport.

Da Betriebe Herrschaftszusammenhänge sind, spielt natürlich auch hier - und zwar ganz zentral - die Beherrschung der Zeit eine wesentliche Rolle. Zeit wird im Betrieb, oder weiter gefasst, in der Ökonomie, in zweierlei Hinsicht beherrscht: als Herrschaft über den Arbeitsprozess - das ist die „Zeitökonomie “ - und als Herrschaft über die Arbeitszeit und ihre Grenzen - das ist die Zeitpolitik.

Zeitökonomie ist die (historisch veränderliche) Art und Weise, wie Zeit „bewirtschaftet", das heißt, in ein Verhältnis zur Wertschöpfung gesetzt wird. Zeitpolitik ist dagegen die Herstellung eines Verhältnisses von ökonomischen und sozialen Zeitstrukturen, von abstrakter (System- oder Betriebszeit) und sozialer Zeit. Zeitpolitik ist damit grundsätzlich beides: der „politische Arm" der Ökonomie und zugleich die Formulierung sozialer Reproduktionsbedürfnisse und sozialer Zeitordnungen. Die Zeitpolitik ist auch der Ort der Auseinandersetzung, des Aufeinandertreffens und/oder der Vermittlung verschiedener Qualitäten von Zeit, vor allem zwischen der zeitökonomischen Qualität, mit möglichst wenig Zeitaufwand möglichst viel zu erreichen (Böhle 1999, S. 14), und der lebensweltlichen Qualität, möglichst viel Zeit für etwas zu haben. Im Fordismus geht es um die Vermittlung zwischen der hierarchisch strukturierten - sozusagen unmittelbar beherrschten - Betriebszeit und der nur mit- telbar beherrschten, deshalb selbstbestimmteren Zeit jenseits des Betriebs. ${ }^{2}$

Bleiben wir jetzt zunächst bei der Zeitpolitik, der Herrschaft über die Arbeitszeit, für die im Fordismus die Stechuhr als Symbol und Funktion paradigmatisch ist. Die Stechuhr markiert - als Funktion - die Grenze zwischen der Sphäre der Produktion und der der Reproduktion. Sie ist zunächst nichts anderes als eine Zeitgrenze, ein Datum. Aber hier werden nicht nur Vorher und Nachher unterschieden, sondern Sphären der gesellschaftlichen Reproduktion voneinander abgegrenzt. Die Stechuhr trennt die Sphären, in denen die Person agiert (als Besitzer und Verkäufer der Ware Arbeitskraft, als Konsument, als Vater oder Mutter, als Vereinsmitglied usw.), von der Sphäre der fremdbestimmten Verausgabung und Nutzung des Arbeitsvermögens, also des Gebrauchswerts der Ware Arbeitskraft. Zugleich wird mit der Stechuhr ein selbstbestimmter Lebensbereich von einem fremdbestimmten Lebensbereich getrennt. In dem Moment, in dem Beschäftigte ihre Karte einstecken, erfüllen sie ihre zentrale vertragliche Anforderung: Sie unterwerfen sich ab einem bestimmten Zeitpunkt für eine bestimmte Zeitstrecke der Herrschaft des Betriebs. Ab diesem Moment und bis zum jeweiligen Ende des Arbeitstages „gehört" die Zeit dem Arbeitgeber bzw. dem Unternehmen. Deshalb ist die Stechuhr auch mehr als nur ein Zeiterfassungsgerät. Sie ist ein Instrument der Transformation gesellschaftlicher Herrschaftsverhältnisse in betriebliche. Und sie ist zugleich ein Symbol für historisch bestimmte gesellschaftliche Herr-

\footnotetext{
Generell steigt mit höherem Qualifikationsniveau der Grad der "Arbeitszeit-Freiheit" (TrautweinKalms 1995) - und genauso generell die Länge der tatsächlichen Arbeitszeit (Kratzer et al. 2005). Bei denjenigen mit formal mehr oder weniger frei gestaltbaren Arbeitszeiten gibt es dennoch große Unterschiede: Sowohl hinsichtlich der Zahl der regelmäßigen Mehrarbeitsstunden wie auch hinsichtlich der Wahrnehmung von „echten “ Gestaltungsspielräumen spielt eine (mit)entscheidende Rolle, wie unmittelbar oder mittelbar die Beschäftigten mit Markt- bzw. Kundenanforderungen konfrontiert sind (Boes/Trinks 2006; Kratzer 2003).

2 Wir verwenden hier einen abstrakten Begriff von Zeitpolitik, der mit Blick auf die kapitalistische Ökonomie sowohl Arbeitszeitpolitik als auch andere Formen gesellschaftlicher Zeitpolitik einschließt. Deren Unterscheidung ist - wie noch zu zeigen sein wird - selbst Resultat der historischen Entwicklung.
} 
schaftsverhältnisse, die ihr Fundament in den Betrieben haben. Was man wann im Betrieb tut, unterliegt nicht der eigenen Entscheidung, sondern folgt den Entscheidungen von Vorgesetzten und/oder den technisch-organisatorischen Zwängen des Produktionsprozesses. Die Stechuhr ist damit auf zwei Ebenen ein Herrschaftsinstrument: als Instrument der zeitlichen Begrenzung von individueller Freiheit und als zeitlicher Rahmen der Ein- bzw. Unterordnung unter einen Herrschaftszusammenhang. Dies erklärt, warum auch die Unternehmen bislang zögern, Vertrauensarbeitszeit auf breiter Front einzuführen oder wenigstens einzufordern. Das für "echte“ Vertrauensarbeitszeit notwendige Vertrauen in die Leistungsbereitschaft der Beschäftigten fehlt vielerorts im Management und bei Führungskräften, im Gegenteil: Es entsteht sogar in neuer Weise Misstrauen (Böhm et al. 2004; Haipeter et al. 2002).

Wenn die Arbeitszeit betrieblich nicht mehr kontrolliert wird und im Zweifel auch gar nicht mehr kontrollierbar ist, ist das unmittelbar ein Zugewinn von Autonomie für die Beschäftigten, zumindest aber eine Rücknahme von Herrschaft. Warum wehren sich Betriebsräte und Gewerkschaften dann gegen diese neue Freiheit oder fordern sogar die Wiedereinführung der Zeiterfassung und Kontrolle?

Eine erste Antwort: Ein Herrschaftsinstrument wie die Stechuhr funktioniert in zwei Richtungen. Die Stechuhr markiert die Grenze von Freiheit, aber sie markiert auch die Grenze von Herrschaft. Die individuelle Freiheit endet am Werkstor, aber auch die betriebliche Herrschaft endet dort. Die Herrschaft der Grenze ist immer auch die Grenze der Herrschaft. Die Stechuhr ist daher nicht nur ein Herrschaftsinstrument, sondern auch ein Instrument, um Herrschaft zu begrenzen. Und damit ist sie das Ergebnis von Zeitpolitik und in ihrer jeweiligen Gestalt ganz wesentlich von gesellschaftlichen Kräfteverhältnissen und gesellschaftlicher Regulierung bestimmt.

Dies erklärt auch, warum Gewerkschaften immer ein ambivalentes Verhältnis zur Stechuhr hatten (Böhm et al. 2004). Einerseits manifestierte sich im Ausdruck „Terror der Stechuhr" die ganze Misere der unfreiwillig verkauften Lebenszeit; darin spiegelten sich auch die vielfältigen Versuche der Unternehmen wider, die gekaufte Zeitspanne möglichst vollständig betrieblich zu nutzen. Andererseits markiert die
Stechuhr zwar die Grenze der individuellen Freiheit, aber eben in beide Richtungen. So konsequent, wie die Lebenszeit mir im Betrieb nicht mehr gehört, so konsequent gehört sie mir „vorher“ und „nachher“. Das schafft einen Rahmen für die betrieblichen Ansprüche an das Arbeits- und Leistungsvermögen, aber es ist zugleich auch die Grenze für diese Ansprüche.

Als geronnenes Ergebnis politischer Kämpfe kommt also in der Zeitpolitik betriebliche Herrschaft und zugleich Gegenmacht zum Ausdruck. Damit wird dann schon etwas klarer, warum jemand etwas gegen die Abschaffung der betrieblichen Kontrolle über die Zeit haben kann: Damit gehen zugleich Möglichkeiten einer politischen Kontrolle von Herrschaft verloren.

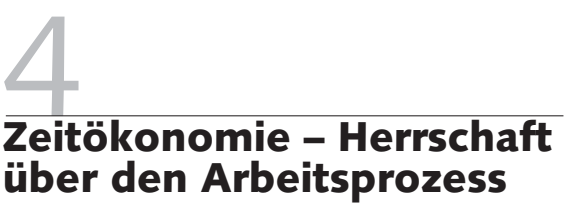

Die Stechuhr als Symbol für die standardisierte, kollektiv regulierte Herrschaft über die Zeit stellt ein Entsprechungsverhältnis her zwischen der betrieblichen Zeitökonomie, den Formen betrieblicher Herrschaft und den sozialen Bedürfnissen mit den darin eingelassenen gesellschaftlichen Herrschaftsverhältnissen. Die Stechuhr ist damit auch Ausdruck eines politisch hergestellten und eine Zeit lang hoch funktionalen Verhältnisses von Ökonomie, Herrschaft und gesellschaftlicher Reproduktion.

Gegenwärtig sind Betriebsräte und Gewerkschaften mit dem Phänomen konfrontiert, dass Beschäftigte von ihren Arbeitszeit-Freiheiten eben gerade nicht Gebrauch machen, sondern sich "freiwillig“ der Fremdherrschaft über ihre Zeit unterwerfen. Ihren plakativen Ausdruck findet diese Entwicklung in den viel zitierten Beispielen von Beschäftigten, die ausstempeln und danach wieder an ihren Arbeitsplatz zurückkehren und weiterarbeiten. Wie ist dieses Phänomen zu interpretieren? Offensichtlich bricht sich die in der Stechuhr symbolisierte, traditionelle, fordistische Zeitpolitik zunehmend an neuen Verhältnissen und "passt" nicht mehr zur historischen Entwicklung.

Ein wesentliches Merkmal dieser Entwicklung ist der Wandel der betrieblichen Zeitökonomie. Sie befindet sich in einem radikalen Umbruch, der hier nur angedeu- tet werden kann. Das dominante Rationalisierungsprinzip der fordistischen Zeitökonomie war die lineare Beschleunigung der Arbeitsprozesse, also zunächst die Beschleunigung der einzelnen Arbeitsprozesse und dann die Optimierung der Schnittstellen zwischen den einzelnen Schritten eines Produktions- und Dienstleistungsprozesses. Dabei ging es vor dem Hintergrund weitreichender technischer und organisatorischer Maßnahmen zur Steigerung der Produktivität immer um die Bewirtschaftung der Zeitmenge, das heißt des Arbeitsaufwandes. Arbeitsorganisatorische Rationalisierung und leistungspolitische Regulierung zielten auf die vollständige Unterordnung der Arbeitszeit unter die Betriebszeit. Die Arbeitszeit wurde von arbeitsfernen Elementen bereinigt. Die Betriebs- und Arbeitsorganisation „kalkuliert(e) zunehmend, mit Hilfe geeigneter Meßmethoden, den einzelnen Arbeiter ebenso nach seinem Rentabilitätsoptimum, wie irgendein sachliches Produktionsmittel“ (Weber 1980, S. 686). Intensive zeitökonomische Rationalisierung kennzeichnet das fordistische Arbeitszeitregime - ebenso wie eine sukzessive Verkürzung der Arbeitszeit. Mit der Eliminierung lebensweltlicher Sinnelemente und spontaner individueller Handlungsorientierungen aus dem Betrieb entsteht mit der Freizeit eine neue Zeitdimension, in der verloren gegangene Freiheitsräume und Lebensbedürfnisse sich neu formieren. Der Preis der Freizeit ist jedoch der Verlust von Autonomie, zeitlicher Gestaltungskraft und Lebensimpulsen in der Arbeit. Damit ging zugleich eine Aufspaltung in verschiedene Politikbereiche einher: Zeitpolitik wurde immer mehr zu Arbeitszeitpolitik, die sich im Wesentlichen mit der Grenze zwischen Arbeits- und Lebenszeit befasste. Demgegenüber ist die Regulierung der Zeit in der Arbeit der zentrale Gegenstand der Leistungspolitik. ${ }^{3}$ Gegenwärtig zeichnet sich die Herausbildung eines diametral anderen Rationalisierungsprinzips ab, das ins-

3 Diese Trennung in zwei Politikbereiche setzt sich auch in den darauf bezogenen Wissenschaftsdisziplinen fort. Für die Forschung zur Arbeitszeit bleibt das zeitökonomische Geschehen häufig eine Art Black Box, das nicht weiter untersuchte "Innere" der Arbeitszeit. Und vice versa bleibt beim Blick auf Leistung und betriebliche Zeitökonomie das Verhältnis zur Lebenswelt meistens stark unterbelichtet. 
besondere durch zwei Merkmale gekennzeichnet ist.

Erstens erfolgt im Zuge von Vermarktlichung, Informatisierung und Globalisierung die Beschleunigung der Arbeitsprozesse nicht mehr nur linear, sondern zunehmend auch synchron. An die Stelle einer Beschleunigung der Prozesse durch lineare Rationalisierung tritt zunehmend der Versuch, Beschleunigung durch Gleichzeitigkeit herzustellen. Synchronisation und Simultanität werden zu den Zielgrößen international organisierter, informatorisch vernetzter Wertschöpfung. Damit gerät aber die Zeitmenge - und damit auch der Arbeitsaufwand - aus dem Fokus betrieblicher Rationalisierung. An die Stelle der Bewirtschaftung der Zeitmenge tritt zunehmend die Bewirtschaftung des Zeitpunkts: Time-to-Market und Just-in-Time sind die Schlagworte dieses Prinzips (Castells 2001; Brose 1994).

Zweitens: Die neue Zeitökonomie organisiert den Verwertungsprozess vom Ende, vom Ergebnis, in letzter Konsequenz vom Markterfolg her. Dies wird am deutlichsten in der gegenwärtigen Entwicklung der Leistungspolitik. Das „Leistungsprinzip“ als Verhältnis von individuellem Aufwand (bzw. von individueller Anstrengung) und Ertrag gerät zunehmend unter den Druck des um sich greifenden „Marktprinzips“. Die Steuerung, aber auch Kontrolle und Bewertung (und am Ende Entlohnung) von Leistung bemisst sich dann tendenziell weniger am Aufwand und mehr am marktbewerteten Ergebnis (Peters/Sauer 2006; Kratzer/Sauer 2004; Menz 2005).

Der richtige Zeitpunkt und das marktbewertete Ergebnis sind somit die Fluchtpunkte der neuen Zeitökonomie. Und genau diese Entwicklung steht einer Zeit- und Leistungspolitik entgegen, die primär die Zeitmenge und den Arbeitsaufwand reguliert. Die Aufwandsorientierung bricht sich an der Ergebnisorientierung - das Ergebnis wird gleichgültig gegenüber dem Aufwand - und die Mengenorientierung bricht sich an der Zeitpunktorientierung.

Das ist eine erste Erklärung dafür, warum Beschäftigte von ihrer neuen „Arbeitszeit-Freiheit" (Trautwein-Kalms 1995) keinen Gebrauch machen können: Ihre Freiheit ist eine der Selbststeuerung des mengenmäßigen Aufwands. Und im gleichen Maße, in dem betriebliche Organisation und Steuerung von Arbeit von der Zeitmenge abstrahiert, ist diese Freiheit nutzlos bzw. verkehrt sich sogar in ihr Gegenteil und führt zur Selbstintensivierung und -extensivierung.

\section{2 \\ Zeitökonomie und Zeitpolitik - Ein neues Herrschaftsverhältnis}

Die Veränderungen der betrieblichen Zeitökonomie sind Teil eines umfassenden gesellschaftlichen Strukturwandels, den wir als einen Prozess der Vermarktlichung charakterisieren und in dem sich eine historisch neue Form der Steuerung von Arbeit herausbildet. Dabei handelt es sich um eine Ablösung des alten Kommandosystems durch ein Herrschaftsprinzip, das wir als Indirekte Steuerung bezeichnen. Der Grundgedanke der Indirekten Steuerung besteht darin, die Form der Abhängigkeit, in der sich der "freie Unternehmer" gegenüber seinen Rahmenbedingungen befindet, zur Steuerung unselbstständig Beschäftigter zu verwenden. „Das Manöver hat zwei Seiten: Erstens reicht die Unternehmensleitung die Umgebungs- und Überlebensbedingungen des Unternehmens (den ,Markt') bis auf den einzelnen Arbeitsplatz durch (statt sie in der Kommandozentrale des Unternehmens abzufangen), und zweitens macht die Unternehmensleitung sich selbst als eine zusätzliche Rahmenbedingung für den abhängig Beschäftigten geltend. Dieses Doppelmanöver versetzt die Arbeitgeber in die Lage, ihre Mitarbeiter während der Arbeitszeit tun zu lassen, was diese selber wollen. Vorausgesetzt allerdings, dass sie ihren Umgebungsbedingungen Genüge tun wie es der selbständige Unternehmer gegenüber seinen Umgebungsbedingungen auch tun muss" (Peters/Sauer 2006, S. 109).

Betriebliche Herrschaft wandelt sich von einem hierarchisch-bürokratischen Anweisungsverhältnis, das sich in bestimmten Akteuren, den Vorgesetzten, in der Zuweisung von Kompetenzen und der Kontrolle von und durch Grenzen konkretisiert, zu „systemischer Herrschaft“. Die „befreiten“ Arbeitskräfte sind jetzt nicht mehr mit konkreten Herrschaftspersonen und konkreten Grenzen ihrer Freiheit konfrontiert, sondern mit dem System selbst, dem Unternehmen und dessen Verwertungsbedingungen. Der Umbruch der Zeitökonomie verschiebt das Objekt der Beherrschung von der kontrollierten Zeit- menge zum kontrollierten Ergebnis. Und die Indirekte Steuerung verschiebt das Subjekt von Herrschaft: Die neue Autonomie der Beschäftigten ist die Freiheit, sich selbst in Auseinandersetzung mit systemischen Anforderungen zu steuern, aus eigenem Willen zum richtigen Ergebnis zu kommen. Die Strafe ist dann nicht mehr die Sanktion, die der Herrschaft zur Verfügung steht, sondern der eigene Misserfolg.

Mit der Indirekten Steuerung verändert sich demnach nicht nur die Zeitökonomie, die Form der Herrschaft über den Arbeitsprozess, sondern auch die Herrschaft über die Zeit, die Zeitpolitik als Form der „Grenzziehung“. Das ganze Verhältnis von Zeitökonomie und Zeitpolitik gerät in Bewegung und damit auch die historischen "Zeitarrangements" - oder, als Herrschaftsverhältnis ausgedrückt: das historische „Zeitregime“ (Deutschmann 1983), das über längere Zeit eine gewisse Stabilität und Kohärenz aufwies. Die Widersprüche zwischen Zeitökonomie und Zeitpolitik waren während dieser historischen Zeitspanne institutionell relativiert. Das meint auch die Rede von einem „industriellen“ oder „fordistischen“ Zeitregime, das eng mit der Entwicklung der fordistischen Formation verknüpft war. ${ }^{4}$ Seine Auflösung führt zu Instabilität und Unsicherheit nicht nur in den Betrieben, sondern auch in den lebensweltlichen Verhältnissen, denn es handelt sich um „Arrangements", in die nicht nur die jeweiligen technisch-organisatorischen Bedingungen, die konkreten Machtverhältnisse und die materiellen Reproduktionsbedürfnisse eingehen. Es geht immer auch um die Besonderheiten einzelner Branchen, Regionen, Kulturen und um die sozialen und emotionalen Bedürfnisse und Anforderungen von Individuen und Familien, die Anforderungen von Konsumenten usw. Die aktuelle Auseinandersetzung um „Zeitkonflikte“ in diversen gesellschaftlichen Bereichen bestätigt den gesellschaftlichen Charakter dieses Umbruchs.

Das ehemals stabile Verhältnis von Zeitökonomie und Zeitpolitik ist aufgebro-

4 Zeitregime dürften ganz wesentlich zur Stabilisierung einer Formation beitragen, weil in ihnen ja entlang einer zentralen gesellschaftlichen Dimension die soziale Reproduktion der Gesellschaft organisiert ist und weil diese Dimension - wie die Geschichte etwa des Kampfes um den Normalarbeitstag zeigt - konfliktträchtig und konfliktfähig ist (Deutschmann 1983). 
chen, die eine Zeit lang stillgelegten Interessenkonflikte werden virulent. Was heißt das nun für die eingangs aufgeworfenen Fragen nach einer „neuen Arbeitszeitpolitik"?

\section{Welche Arbeitszeitpolitik?}

\subsection{FLEXIBILISIERUNG - DIE ANPASSUNG AN DIE NEUE ZEITÖKONOMIE}

Mit dem Wandel der Zeitökonomie geht der traditionellen Arbeitszeitpolitik zunehmend ihr Gegenstand (Zeitmenge bzw. -aufwand) verloren. Mit der Indirekten Steuerung erweisen sich die traditionellen Formen der Steuerung und Kontrolle von Zeit als weitgehend unwirksam. Die vielfältigen Formen einer Flexibilisierung von Arbeitszeit erscheinen vor diesem Hintergrund eher als Anpassung der Zeitpolitik an den Wandel der Zeitökonomie denn als Beginn einer „nachfordistischen Ära“ der Arbeitszeitpolitik. Hatte der Kampf um die Verkürzung der Arbeitszeit, der ja den Kern der fordistischen Zeitpolitik bildete, noch den Charakter einer politischen Auseinandersetzung „um die Herrschaft über die Zeit", so erscheint die Flexibilisierung der Arbeitszeit eher als eine Durchsetzung „ökonomischer Sachzwänge“, die dann mühsam in einigermaßen regulierte Formen gegossen werden (können). Dabei sind durchaus Veränderungen in den kollektiven Regulierungsmustern zu erkennen: Die neuen Regelungen sind indirekter - sie beziehen sich mehr auf Verfahren und weniger auf Inhalte - und vielfach auch individueller und partizipativer. Es erscheint jedoch fraglich, ob mit der ,kontrollierten Flexibilität" bereits ein neuer Steuerungsmodus entstanden ist. Unserer Einschätzung nach handelt es sich eher um eine Modifikation vorhandener Kontrollformen, die den realen Verlust von Einfluss nicht verhindern kann. Mit den Auseinandersetzungen um die Flexibilisierung der Arbeitszeit konnten die Gewerkschaften und Betriebsräte bislang weder die angestrebten „Machtrückgewinne“ realisieren noch das durchaus vorhandene neue Potenzial für individuelle „Zeitsouveränität“ nutzen.

Während sich für die Unternehmen die Freigabe der Kontrolle über die Arbeitszeit nicht als Verlust an Herrschaft, sondern als Wandel der Form von Herrschaft darstellt (von der „personalen“ zur „systemischen“ Herrschaft), bleibt auf der Arbeitnehmerseite der Verlust von Einfluss auf die Bestimmung und Kontrolle von Arbeitszeitgrenzen. Die Rede von der arbeitspolitischen Defensive oder vom Machtverlust der Gewerkschaften hat hier ihre wesentlichen Ursachen.

\subsection{DAS HANDLUNGSDILEMMA GEWERKSCHAFTLICHER ARBEITSZEITPOLITIK}

Aus der Sicht von Gewerkschaften und Betriebsräten ist es jedoch zunächst nur allzu verständlich, an den noch vorhandenen Einflussmöglichkeiten auf die „Herrschaft über die Zeit" festzuhalten. Der Doppelcharakter der „Herrschaft über die Zeit" am Beispiel der Stechuhr, wie wir ihn oben beschrieben haben, macht die ambivalente Rolle von Instrumenten der Zeitkontrolle deutlich. Allerdings war die Schutzfunktion solcher Instrumente - als Grenze unternehmerischer Herrschaft - für die Beschäftigten daran gebunden, dass auf der Arbeitnehmerseite eine politische Instanz fungiert, die die individuellen Interessen der Beschäftigten stellvertretend wahrnimmt - also an die korporatistische Struktur von Interessenvertretung. Gegenwärtig wird sichtbar, dass mit der Auflösung des fordistischen Zeitregimes auch dieses Element zunehmend erodiert. Im Verlust der Verhandlungsmacht von Betriebsräten und Gewerkschaften wird dies deutlich. Hier liegt das eigentliche Dilemma gewerkschaftlicher Arbeitszeitpolitik: Gewerkschaften und Betriebsräte sind auf der einen Seite gezwungen, im verbleibenden Rahmen fordistischer Zeitpolitik zu agieren und ihre Schutzfunktion wahrzunehmen. Sie geraten dabei gleichzeitig in den Strudel der Auflösung dieser Politik - und damit vielfach in Gegensatz zu den Beschäftigten, deren Interessen sie vertreten sollen. Ihre Befangenheit im fordistischen Zeitregime verhindert auf der anderen Seite einen offeneren Umgang mit zeitpolitischen Konflikten und erschwert die Entwicklung neuer politischer Bewegungsformen jenseits eingeübter Verfahrensweisen. Aber was heißt „neue politische Bewegungsformen"? Was sind die Anforderungen an eine neue Arbeitszeitpolitik, die den historischen Veränderungen gerecht wird?

\subsection{INDIVIDUALISIERTE ARBEITSZEITPOLITIK}

Ausgangspunkt für Antworten auf diese Fragen könnte der theoretische Grundgedanke sein, dass Arbeitszeitpolitik im Kapitalismus sich immer auf das Verhältnis von ökonomischen und sozialen Zeitstrukturen bezieht, also verschiedene Qualitäten von Zeit vermitteln muss - ökonomische und lebensweltliche. Fordistische Zeitpolitik hat diese Aufgabe durch die Definition klarer institutioneller Grenzziehungen zwischen arbeits- und lebensweltlichen Zeitstrukturen ,gelöst“. Die veränderte historische Situation verweist aber gerade auf die Entgrenzung und zunehmende Verschränkung der beiden Sphären (Kratzer/ Sauer 2007). Diese Verschränkung ist in beiden Richtungen, als „Kolonialisierung der Lebenswelt“ oder als „Rückkehr des Subjekts in die Ökonomie“, vielfach beschrieben und auch empirisch belegt worden. Unklar und politisch ungelöst ist die Frage, wie die weiterhin notwendigen Grenzziehungen zwischen den beiden Sphären nun erfolgen sollen. Offensichtlich verlagern sich die Vermittlung und damit auch die Grenzziehung zunehmend ,in die Individuen selbst".

Diese Perspektive spitzt sich zu, wenn ein zweiter theoretischer Grundgedanke berücksichtigt wird: Die Ablösung betrieblicher Zeitökonomie von Arbeitsmenge und Arbeitsaufwand, die zunehmende Zeitpunkt- und Ergebnisorientierung lässt bislang gültige normative Maßstäbe menschlicher Leistungsfähigkeit in den Hintergrund treten (Sauer et al. 2004). Sie verlieren einerseits ihre Schutzfunktion vor allem dann, wenn gleichzeitig die Grenze von Arbeits- und Lebenszeit durchlässig wird. Andererseits wird dadurch der Zwang zur Grenzziehung für den einzelnen Beschäftigten zur existenziellen Lebensfrage. Der in der betrieblichen Zeit- und Leistungspolitik verschwundene Aufwandsbezug macht sich im individuellen Umgang mit Zeit umso drängender und unausweichlicher bemerkbar. Die gegenwärtig an vielen Stellen sichtbare Zunahme psychischer Belastungen und daraus resultierender Krankheiten sind ein Indikator für steigenden Leistungsdruck und individuelle Zeitnot. Die Verlagerung der Grenzziehung ins Individuum bedeutet jedoch nicht die Stilllegung des Kampfes um die „Herrschaft über die Zeit“. Die latente Konfliktstruktur bricht an vielen Stellen auf, ohne 
dass bislang "gerichtete" Formen eines Widerstandes zu erkennen wären. Die Konfrontation mit den Rahmenbedingungen Indirekter Steuerung erzwingt die Auseinandersetzung des Individuums mit sich selbst. Lässt es sich und sein Leben von den Rahmenbedingungen bestimmen oder entwickelt es ein selbstbestimmtes Verhalten gegenüber den Rahmenbedingungen? In dieser Auseinandersetzung die Beschäftigten nicht alleine zu lassen, sondern Räume der Verständigung zu schaffen und Formen der gemeinsamen Interessenidentifikation zu unterstützen, wäre eine erste und zentrale Voraussetzung für eine Neuausrichtung von Arbeits(zeit)politik.

\subsection{KONFRONTATION MIT DER ZEITÖKONOMIE}

Dass in solchen Situationen der individuellen Überforderung der Wunsch nach der Rückkehr alter Formen der „Herrschaft über die Zeit" laut wird, ist nicht verwunderlich. Dennoch ist den betroffenen Beschäftigten meist sehr klar, dass sie damit das Problem nicht lösen würden. Sie müssen ihre Aufgaben erledigen, Termine einhalten und Ergebnisse erbringen. Stechuhren oder ähnliche Instrumente der Zeitkontrolle bieten dabei keinen Schutz mehr.

Die betriebliche Herrschaft über die Zeit ist sehr viel stärker in die neue zeitökonomische Struktur der Arbeitsprozesse eingelagert. Nicht einzelne betriebliche Akteure, nicht hierarchische Instanzen oder Vorgesetzte, sondern in den Arbeitsablauf integrierte Stellgrößen (Termine, Kundenanforderungen, vorhandenes Personalvolumen, Zugriff auf Ressourcen, verfügbare Qualifikationen und nicht zuletzt in Kennziffern gegossene Rendite-Erwartungen) entscheiden über die zeitlichen Arbeitsanforderungen und deren Wirkungen. Zeitpolitische Veränderungen sind darauf angewiesen, Einfluss auf diese Stellgrößen zu nehmen.

Damit steht nichts Geringeres an als eine Umkehrung der fordistischen Zeitpolitik. Der Kampf um Arbeitszeitverkürzung war der Kampf um mehr Freiheit jenseits der Arbeit, das Mittel war die strikte Grenzziehung zwischen „Arbeit und Leben“. Und in der Kontrolle dieser Grenze durch politische Regulierung war die Gegenmacht der Arbeitnehmer und ihrer Interessenvertretung institutionalisiert. Der Preis für die Verschiebung dieser Grenze, für die Aus- dehnung der „freien Zeit“, war der zunehmende Verlust von Kontrolle über die Arbeit, die Unterwerfung unter das kommandoförmige fordistische Zeitregime.

Mit dem Wandel der Zeitökonomie, der Auflösung der Grenzen zwischen Arbeit und Leben erscheint politische Gegenmacht allein durch die Regulierung und Kontrolle von Grenzen nicht mehr möglich, weil sie praktisch zunehmend unwirksam wird. Zeitökonomische Durchdringung erfasst das Leben außerhalb der Betriebe und holt subjektive und lebensweltliche Qualitäten in die Arbeit zurück. Indirekte Steuerung setzt auf die Nutzung der individuellen Autonomie der Beschäftigten und deren Selbststeuerung und Selbstorganisation von Arbeitsprozessen. Entscheidende Steuerungsgrößen für die Unternehmen liegen darin, Rahmenbedingungen zu setzen. Diese geraten nun ins Kampffeld der Auseinandersetzung um die „Herrschaft über die Zeit", denn sie entscheiden - indirekt - über die „Zeitgrenzen“ der Beschäftigten.

Der Kampf um die Zeit wird zum Kampf um die Determinanten des Arbeitsprozesses und um dessen Gestaltung. Zeitpolitik wird integraler Bestandteil von Arbeitspolitik - einer Arbeitspolitik, die sich nicht mehr darauf beschränken kann, die Auswirkungen auf die Beschäftigten abzufedern, sondern sich in die Organisation der Rahmenbedingungen von Arbeit einmischen muss, wenn sie Wirkung erzielen will. Und dieser Einfluss muss im Arbeitsprozess von den Beschäftigten selbst inhaltlich definiert und getragen werden. Er lässt sich nicht ohne Bezug zu den individuellen Grenzsetzungen, zu den individuellen Maßstäben durchsetzen. Eine von außen herangetragene Einflussnahme erweist sich ohne Beteiligung der einzelnen Beschäftigten als nicht umsetzbar. Auf der anderen Seite gerät der Kampf um die „Herrschaft über den Arbeitsprozess“ sehr schnell an die Grundfesten kapitalistischer Herrschaft. Umkämpft werden Einflussgrößen, die bislang im Direktionsrecht der Unternehmensleitungen liegen. Die Nähe zu unternehmerischen Kernkompetenzen macht den Konflikt politisch brisant und schwierig. Er kann deswegen letztlich nur in kollektiven Formen im Betrieb und in der Gesellschaft ausgetragen werden. Daraus ergibt sich ein neues Verhältnis von individueller und kollektiver Interessenspolitik. Entscheidend wird es sein, neue produktive Verbindungen von individuellem und kollektivem Interessenhandeln zu finden und weiterzuentwickeln.

\section{Resumee: Arbeitszeit- \\ Resümee: Arbeitszeit- politik im Übergang}

Die Regulierung von Arbeitszeitgrenzen als Kern der klassischen Arbeitszeitpolitik wird zunehmend wirkungslos. Alternative Formen einer „neuen Arbeitszeitpolitik“ sind nicht in Sicht. Die Arbeitzeitpolitik befindet sich weiterhin in einem historischen Übergangsprozess, der immer noch durch die eingangs formulierten Pole - Stechuhr und Vertrauensarbeitszeit - charakterisiert wird. Dazwischen liegt das weite und vielschichtige Feld der Flexibilisierung der Arbeitszeit. Bei der Regulierung von flexiblen Arbeitszeitformen wird, trotz einiger Erfolge, in der Perspektive einer „Begrenzung der Herrschaft über die Zeit" keine neue Qualität erreicht. Wir haben es mit einer weitgehend defensiven Kontrolle der Folgeprobleme zu tun, die die Ausweitung unternehmerischer Herrschaft durch Flexibilisierung mit sich bringt. Je weiter die Auflösung der Arbeitszeitgrenzen geht - bis hin zur Vertrauensarbeitszeit -, desto schwieriger wird die Anpassung der Kontroll- und Regulierungsmöglichkeiten. Die Schlussfolgerungen aus unserer Argumentation beschränken sich nicht auf das empirische Feld der Vertrauensarbeitszeit, die uns nur exemplarisch als Fluchtpunkt für die Analyse der historischen Entwicklung der Arbeitszeitorganisation gedient hat. Die Erosion der traditionellen Arbeitszeitpolitik hat viele Facetten und findet sich in vielen empirischen Arbeitzeitformen - uns ging es um den grundsätzlichen Charakter, um die innere Logik des gegenwärtig stattfindenden Umbruchs.

Betriebräte und Gewerkschaften stehen vor einer historischen Aufgabe: Mit dem Wandel betrieblicher Zeitökonomie und der Durchsetzung Indirekter Steuerungsformen entgleitet die frühere objektive Basis für eine Zeitpolitik „der Grenzkontrolle“. Sie erfahren dies einerseits als deutlichen Machtverlust, andererseits bleibt ihnen kaum etwas anderes übrig, als zu versuchen, im Rahmen der noch vorhandenen alten Regulierungsmuster das "Neue“ einzufangen.

Der Übergang von einer stellvertretenden Politik der Regulierung und Kontrolle 
von Zeitgrenzen zur offensiven Auseinandersetzung um den Einfluss auf die Determinanten des Arbeitsprozesses ist ein mühsamer Weg. Er führt letztlich zur Überwindung von Arbeitszeitpolitik als isolierter Politikform: Sie wird zum Bestandteil einer integrierten Arbeitspolitik, in der die Herr- schaft über den Arbeitsprozess in den Fokus gerät. Diese Aufgabe setzt die Mobilisierung bislang schwer zugänglicher individueller Interessen voraus. Das ist ein in besonderer Weise konfliktreicher Weg, denn es liegt in der Natur der Sache, nämlich des Formwandels betrieblicher Herrschaft und der neuen Qualität betrieblicher Zeitökonomie, dass diese Auseinandersetzungen den "Systemfragen“ kapitalistischer Herrschaft und zugleich den individuellen Interessen und Bedürfnissen der Beschäftigten näher auf den Leib rücken.

\section{LITERATUR}

Bauer, F./Groß, H./Lehmann, K./Munz, E. (2004): Arbeitszeit 2003. Arbeitzeitgestaltung, Arbeitsorganisation und Tätigkeitsprofile, Düsseldorf Boes, A./Trinks, K. (2006): „Theoretisch bin ich frei“. Interessenhandeln und Mitbestimmung in der IT-Industrie, Berlin Böhle, F. (1999): Entwicklungen industrieller Arbeit und Arbeitszeit. Umbrüche in der zeitlichen Organisation von Arbeit und neue Anforderungen an den Umgang mit Zeit, in: Büssing, A./Seifert, H. (Hrsg.): Die "Stechuhr" hat ausgedient. Flexiblere Arbeitszeiten durch technische Entwicklungen, Berlin, S. 13-26

Böhm, S./Herrmann, C./Trinczek, R. (2004): Herausforderung Vertrauensarbeitszeit. Zur Kultur und Praxis eines neuen Arbeitszeitmodells, Berlin

Brose, H.-G. (1994): Dimensionen einer reflexiven Ökonomie der Zeit, in: Beckenbach, N./Treeck, W. (Hrsg.): Umbrüche gesellschaftlicher Arbeit, Soziale Welt, Sonderband 9, Göttingen, S. 209-226

Castells, M. (2001): Das Informationszeitalter, Teil 1: Der Aufstieg der Netzwerkgesellschaft, Opladen

Deutschmann, C. (1983): Systemzeit und soziale Zeit, in: Leviathan 4, S. 494-514

Haipeter, T./Lehndorff, S./Schilling, G./Voss-Dahm, D./Wagner, A. (2002): Vertrauensarbeitszeit - Analyse eines Rationalisierungskonzepts, in: Leviathan 3, S. 360-383

Hoff, A. (o. J.): Vertrauensarbeitszeit: einfach flexibel arbeiten, www.arbeitszeitberatung.de/dateien/publikationen/pdf/HoffVertrauensarbeitszeit.exe

Kratzer, N. (2003): Arbeitskraft in Entgrenzung. Grenzenlose Anforderungen, erweiterte Spielräume, begrenzte Ressourcen, Berlin
Kratzer, N./Fuchs, T./Wagner, A./Sauer, D. (2005): Zeitmuster - Zeitverwendung im Kontext von Erwerbsarbeit und Haushalt, in: SOFI/IAB/ INIFES/ISF (Hrsg.): Berichterstattung zur sozioökonomischen Entwicklung in Deutschland: Arbeit und Lebensweisen, Erster Bericht, Wiesbaden, S. 381-402

Kratzer, N./Sauer, D. (2004): Zeit, Leistung, Beschäftigung. Anforderungen an eine erweiterte Arbeits(zeit)politik, in: Seifert, H. (Hrsg.): Flexible Zeiten in der Arbeitswelt, Frankfurt/New York, S. 244-259

Kratzer, N./Sauer, D. (2007): Entgrenzte Arbeit - Gefährdete Reproduktion. Genderfragen in der Arbeitsforschung, in: Aulenbacher, B./Funder, M./Jacobsen, H./Völker, S. (Hrsg.): Arbeit und Geschlecht im Umbruch der modernen Gesellschaft, Forschung im Dialog, im Erscheinen.

Menz, W. (2005): Ende der Leistungsgerechtigkeit? Neue Arbeitspolitik und Beschäftigtenorientierungen, in: WSI-Mitteilungen 2, S. 69-75 Peters, K./Sauer, D. (2006): Epochenbruch und Herrschaft - Indirekte Steuerung und die Dialektik des Übergangs, in: Scholz, D. et al. (Hrsg.).: Turnaround? Strategie für eine neue Politik der Arbeit, Münster Sauer, D. (2005): Arbeit im Übergang. Zeitdiagnosen, Hamburg Sauer, D./Döhl, V./Kratzer, N./Marrs, K. (2004): Arbeiten ohne (Zeit-)Maß? - Ein neues Verhältnis von Arbeitszeit- und Leistungspolitik, in: Bsirske, F./Mönig-Raane, M./Sterkel, G./Wiedemuth, J. (Hrsg.): Es ist Zeit - Das Logbuch für die ver.di-Arbeitszeitinitiative, Hamburg, S. 155177

Trautwein-Kalms, G. (1995): Ein Kollektiv von Individualisten. Interessenvertretung neuer Beschäftigtengruppen, Berlin

Weber, M. (1980): Wirtschaft und Gesellschaft. Grundriss der verstehenden Soziologie, Tübingen 